\title{
The effects of combined bromocriptine and Bu-shen-zhu-yun decoction on serum hormones, anxiety, and pregnancy in hyperprolactinemic infertility patients
}

\author{
Hua Feng ${ }^{1,2 \# \wedge}$, Qiuxi Zhong ${ }^{2 \#}$, Huifang Zhou ${ }^{3}$, Xiaoyue Jiang ${ }^{2}$, Yinyin Ding ${ }^{2}$ \\ ${ }^{1}$ Institute of Rehabilitation, Jiangsu Vocational College of Medicine, Yancheng, China; ${ }^{2}$ Department of Gynecology, Nanjing University of Chinese \\ Medicine, Nanjing, China; ${ }^{3}$ Department of Gynecology, Affiliated Hospital of Nanjing University of Chinese Medicine, Nanjing, China \\ Contributions: (I) Conception and design: H Feng, Q Zhong; (II) Administrative support: H Zhou; (III) Provision of study materials or patients: H \\ Zhou; (IV) Collection and assembly of data: H Feng, Q Zhong; (V) Data analysis and interpretation: X Jiang, Y Ding; (VI) Manuscript writing: All \\ authors; (VII) Final approval of manuscript: All authors. \\ \#These authors contributed equally to this work and should be considered as co-first authors. \\ Correspondence to: Hua Feng. Institute of Rehabilitation, Jiangsu Vocational College of Medicine, Yancheng, China. Email: 20195059@njucm.edu.cn; \\ Huifang Zhou. Department of Gynecology, Affiliated Hospital of Nanjing University of Chinese Medicine, Nanjing, China. \\ Email: zhouhuifang2011301@163.com.
}

Backgroundz Prolactin (PRL) is a protein hormone secreted by the anterior pituitary gland that regulates
pituitary hormones. Hyperprolactinemia (HPRL), a pathological phenomenon of excessive PRL, can cause
infertility in severe cases and is currently treated mainly with Western drugs, such as bromocriptine, a
dopamine agonist (DA). Unfortunately, DAs produce psychological side effects which limit their long-term
use. Traditional Chinese medicine (TCM) has minimal side effects and good results spanning many years
of research. The combined treatment of TCM and Western medicine may enhance treatment efficacy and
improve the long-term prognosis in HPRL. To analyze the effects of Bu-shen-zhu-yun decoction (BSZY-D)
combined with bromocriptine on serum hormones, anxiety, and pregnancy in hyperprolactinemic infertile
patients.

Methods: One hundred patients diagnosed with HPRL infertility from June 2020 to June 2021 in the gynecology clinic of Jiangsu Provincial Hospital of Traditional Chinese Medicine were selected and grouped by envelope method. After excluding patients who withdrew or missed visits, 37 cases assigned to the control group were treated with bromocriptine, and 40 cases assigned to the observation group were treated with bromocriptine combined with BSZY-D. The patients' PRL and kisspeptin (KP) serum indexes, improvements in infertility, Anxiety Self-Assessment Scale (SAS) scores, and improvements in the Insomnia Severity Index Scale (ISI) scores were compared between the two groups.

Results: At 3 and 6 months of treatment, serum PRL, SAS, and ISI scores were significantly lower, and serum KP was significantly higher in the observation group than in the control group $(\mathrm{P}<0.05)$. During the study period, the pregnancy rates were $62.50 \%(25 / 40)$ and $37.84 \%(14 / 37)$ in the observation and control groups, respectively. The observation group also had significantly fewer early miscarriages [10.00\% (4/40) vs. $32.43 \%(12 / 37)]$ and less adverse reactions [7.50\% (3/40) vs. $24.32 \%(9 / 37)]$ than the control group (all $\mathrm{P}<0.05)$.

Conclusions: The combination of bromocriptine with BSZY-D was superior to bromocriptine alone in treating HPRL and HPRL-related infertility, which also demonstrated a positive effect on patients' sleep and low mood.

Keywords: Bu-shen-zhu-yun decoction (BSZY-D); bromocriptine; hyperprolactinemia (HPRL); infertility; sleep

\footnotetext{
^ ORCID: 0000-0003-0942-411X.
} 
Submitted Sep 24, 2021. Accepted for publication Nov 18, 2021.

doi: 10.21037/apm-21-3111

View this article at: https://dx.doi.org/10.21037/apm-21-3111

\section{Introduction}

Hyperprolactinemia (HPRL), the excess of prolactin (PRL), is a common and challenging disease in gynecology, usually producing reproductive problems in both sexes particularly anovulatory infertility in women. The mechanism of HPRL is complex and is usually considered to be caused by a combination of internal and external factors such as pathology, drugs, and organic heterogeneity. Hence, HPRL can be classified into two subtypes based on etiopathogenesis: organic and functional HPRL. HPRL mainly manifests as elevated serum PRL, negatively affecting patients' reproductive functioning and central nervous system. In mild cases, it may lead to amenorrhea, low menstrual flow, and reduced sexual function, resulting in a lower quality of life. In severe cases, it leads to infertility, which may negatively impact the patient's psychological health and family harmony. The main investigations in the diagnosis of HPRL are hormonal and radiological.

Bromocriptine is one of the first-choice drugs for HPRL, and it has a good control effect on serum PRL and sex hormones (1). However, results from related studies (2) show that bromocriptine can cause serious adverse effects, such as nausea and vomiting, vertigo, and, in severe cases, upright hypotension, cardiac arrest, and vasospasm of the lower limbs. Some patients have also reported hallucinations and delusions. These adverse side-effects of bromocriptine lead to a further decrease in patient compliance and limit long-term treatment (3). In contrast, traditional Chinese medicine (TCM) treatment considers the symptoms and root cause and has reliable efficacy and fewer adverse effects. Thus, the combination of bromocriptine and TCM is feasible. Bu-shen-zhu-yun decoction (BSZY-D) is a common formula used for kidney deficiency-type infertility. It has shown good therapeutic results in various infertility disorders, such as ovulation disorder and polycystic ovary syndrome $(4,5)$. Still, there are very few studies on the combined use of BSZY-D with bromocriptine for infertility treatment. Therefore, we conducted a comparative study of 87 patients to observe the effect of combined bromocriptine and BSZY-D on improving mental status, infertility, and HPRL-related serum indicators of HPRL patients, aiming to provide new options for the treatment of HPRL-infertile patients. We present the following article in accordance with the STROBE reporting checklist (available at https:// dx.doi.org/10.21037/apm-21-3111).

\section{Methods}

\section{General information}

All procedures performed in this study involving human participants were in accordance with the Declaration of Helsinki (as revised in 2013). The study was approved by the Ethics Committee of Jiangsu Provincial Hospital of Traditional Chinese Medicine (also named Affiliated Hospital of Nanjing University of Chinese Medicine, No. 2108NL-030-02) and informed consent was taken from all the patients. 100 patients diagnosed with HPRL infertility from June 2020 to June 2021 at the gynecology clinic were selected and grouped by the envelope method. After excluding patients who withdrew, missed visits, or had serious adverse reactions, a total of 37 cases were assigned to the control group, and 40 cases were assigned to the observation group, with the following diagnostic criteria: (I) Western medicine diagnostic criteria: (i) an absence of pregnancy for 12 months without contraceptive sex; (ii) a prolactin (PRL) level $>25 \mu \mathrm{g} / \mathrm{L}$ (6). (II) TCM diagnostic criteria: (i) primary or secondary infertility; (ii) syndrome of depression of heart or liver QI; (iii) menstrual disorder with dark red color; (iv) galactorrhea (v) premenstrual abdominal distension and pain; (vi) tinnitus and dizziness; (vii) frequent sighing; (viii) indifferent sexual desire; (ix) breast distension and pain. Additionally, a light tongue color with thin white coating and string or sunken string pulse. The criteria were satisfied if patients met criteria $1 \mathrm{a}$ and $1 \mathrm{~b}$, two or more criteria from $2 \mathrm{c}-2 \mathrm{i}$, and the pulse-taking (7).

The inclusion criteria were as follows: (I) individuals aged 20-40 years; (II) patients who met the diagnostic criteria; (III) those who signed the informed consent and cooperated with the study throughout (8).

Patients were excluded if they had any of the following conditions: (I) neurological diseases; (II) organic diseases such as pituitary tumors; (III) other diseases causing infertility, such as premature ovarian failure; (IV) elevated serum PRL due to other causes (exercise, hypoglycemia, etc.) (9). 


\section{Medications}

Patients in both groups took bromocriptine (Novartis Farma SPA, Italy; approval number: H20160030; specification: $2.5 \mathrm{mg}^{*} 30 \mathrm{~s}$ ) after menstruation at a dose of $2.5-3.75 \mathrm{mg} /$ day, gradually increasing to $10-20 \mathrm{mg} /$ day in $2-3$ doses, which could only be increased after the diagnosis of the patient's disease by the physician. Patients were advised not to increase or decrease the dose by themselves. In the event of a serious adverse reaction, patients were asked to contact their physician promptly to decide whether to reduce the dosage or suspend the medication.

BSZY-D was added to the observation group's medication regime and consisted of $10 \mathrm{~g}$ of deer horn slices (first decoction), $10 \mathrm{~g}$ of violet quartz (first decoction), $20 \mathrm{~g}$ of dens draconis (first decoction), $12 \mathrm{~g}$ of fried white peony root, $18 \mathrm{~g}$ of dioscorea rhizoma, $9 \mathrm{~g}$ of vinegared radix bupleuri, $10 \mathrm{~g}$ of purple salvia miltiorrhiza, $10 \mathrm{~g}$ of gambir plant (finally add), $18 \mathrm{~g}$ of cuscutae semen, and $12 \mathrm{~g}$ of corni fructus, Add $300 \mathrm{~mL}$ of water and simmer with $100 \mathrm{~mL}$ of water. An amount of $300 \mathrm{~mL}$ of water was added, $100 \mathrm{~mL}$ was decocted and poured out, and then a further $300 \mathrm{~mL}$ of clear water was added and decocted to $100 \mathrm{~mL}$. The decoction was mixed and taken orally twice daily (morning and evening) but suspended during menstruation. The dosage and course of BSZY-D treatment in the present study were based on the previous studies $(4,5)$.

In both groups, the prescribed medication was taken continuously for 3 months, during which time other medications were forbidden. If menstruation did not occur for more than 1 week, patients were advised to have a pregnancy test promptly, and if pregnancy was confirmed, the medication was stopped.

\section{Observed indicators}

The following indicators were compared between the two groups of patients:

(I) Baseline information (age, gestation, etc.).

(II) Before treatment, and at 3 and 6 months of treatment, serum PRL and KP indicators were assessed. An amount of $3 \mathrm{~mL}$ of venous blood was collected from patients between 9 and 10 am on the $3 \mathrm{rd}$ or 4th day of menstruation on an empty stomach and centrifuged after standing at room temperature. Patients were required to sit for 15 min before collection. The serum PRL was detected by electrochemiluminescence immunoassay
(Roche Cobase 601), and serum KP was detected by ELISA assay [human kissin (Kp) enzyme-linked immunoassay kit; Jianlai Bio, Shanghai, China].

(III) Anxiety and sleep assessments:

The Anxiety Self-Assessment Scale (SAS) (10) is a 20 -item self-report assessment with possible total scores from $20-80$. Scores are rated as $<50=$ normal, $50-60=$ mild, $61-70=$ moderate, and $>70=$ severe. Individual items within the SAS were compared between the two groups (e.g., "I feel more nervous and anxious than usual", "I feel worried and scared for no reason", "I get upset or panic easily", and "I feel like I might be going crazy").

The Insomnia Severity Index (ISI) (11) is a 7-item assessment of sleep quality and insomnia, where scores between $0-7=$ nil, $8-14=$ mild, $15-21=$ moderate, and 22-28= severe insomnia. Individual items within the ISI were compared between the two groups (e.g., difficulty in falling asleep, difficulty in maintaining sleep, problems with waking up too early, satisfaction with sleep status).

(IV) Pregnancy rate, serum HCG to determine pregnancy, and diagnosis of pregnancy by the intrauterine sighting of the fetal heart.

(V) Adverse reactions, including hematoma of the nostrils, headache and dizziness, nausea and regurgitation, severe constipation, or increased anxiety.

\section{Statistical methods}

SPSS v.22.0 software was used to analyze the data. Measurement data are expressed as the mean $\pm \mathrm{SD}(\bar{x} \pm \mathrm{s})$, and the Student's $t$-test was used to compare the means between groups. Categorical count data are expressed as percentages (\%), and the $\chi^{2}$ test was used to compare between-group differences. A $\mathrm{P}$ value $<0.05$ was considered statistically significant.

\section{Results}

\section{Comparison of baseline information between the two patient groups}

The results of the comparison showed that there was no significant difference in the baseline information of age, annual income, type of infertility, duration of education, or career between the two groups $(\mathrm{P}>0.05)$, allowing a comparative study to be conducted (Table 1). 
Table 1 Comparison of baseline information between the two patient groups [n, (\%)]

\begin{tabular}{|c|c|c|c|c|}
\hline Groups & Observation group $(n=40)$ & Control group $(n=37)$ & $t$ & $P$ \\
\hline Regular occupation & $17(42.50)$ & $16(43.24)$ & & \\
\hline No regular occupation & $14(35.00)$ & $13(35.14)$ & & \\
\hline Unemployed & $9(22.50)$ & $8(21.62)$ & & \\
\hline$\leq 9$ & $6(15.00)$ & $5(13.51)$ & & \\
\hline $9-12$ & $18(45.00)$ & $17(45.95)$ & & \\
\hline $12+$ & $16(40.00)$ & $15(40.54)$ & & \\
\hline Age & $30.93 \pm 5.08$ & $31.08 \pm 5.14$ & 0.129 & 0.900 \\
\hline $50-100,000$ & $15(37.50)$ & $14(37.84)$ & & \\
\hline $100-200,000$ & $9(22.50)$ & $9(24.32)$ & & \\
\hline$\geq 200,000$ & $5(12.50)$ & $4(10.81)$ & & \\
\hline Type of infertility & & & 0.030 & 0.862 \\
\hline Primary infertility & $17(42.50)$ & $15(40.54)$ & & \\
\hline Secondary infertility & $23(57.50)$ & $22(59.46)$ & & \\
\hline
\end{tabular}

Comparison of serum PRL and serum KP between the two patient groups

Before treatment, the comparison of serum PRL and serum KP between the observation and control groups showed no statistically significant difference $(\mathrm{P}>0.05)$. At 3 and 6 months of treatment, serum PRL had decreased in both groups but was significantly lower in the observation group than in the control group. Serum KP was elevated in both groups but was significantly higher in the observation group than in the control group $(\mathrm{P}<0.05)($ Table 2$)$.

\section{Comparison of SAS and ISI scores between the two patient groups}

Before treatment, the comparison of SAS and ISI scores in the observation and control groups showed no statistically significant differences $(\mathrm{P}>0.05)$. At 3 and 6 months of treatment, SAS scores and ISI scores had decreased in both groups, but at both time points, the observation group's scores were significantly lower than those of the control group $(\mathrm{P}<0.05)$. Of the 20 SAS individual items, 14 items were statistically significant between the two groups at 3 and 6 months $(\mathrm{P}<0.05)$, and six items were not statistically significant $(\mathrm{P}>0.05)$ ("I have to urinate frequently", "I am suffering from stomach pain and indigestion distress", "my fingers and toes felt numb", "I had bouts of feeling like I was going to faint", "I felt uncomfortable due to bouts of vertigo", "my limbs shook and trembled"). Of the 7 ISI symptoms, except for the two indicators related to difficulty in falling asleep and difficulty in maintaining sleep, all other indicators were statistically different between the two groups after 6 months of treatment $(\mathrm{P}<0.05)$ (Tables 3,4).

\section{Comparison of pregnancy, early miscarriage, and adverse reaction rates between the two groups}

During the study period, there were 25 and 14 successful pregnancies, 4 and 12 early miscarriages, and 3 and 9 cases of adverse reactions in the observation and control groups, respectively, with significant differences between the groups $(\mathrm{P}<0.05)($ Table 5). 
Table 2 Comparison of serum PRL and serum KP between the two groups $(\bar{x} \pm \mathrm{s})$

\begin{tabular}{|c|c|c|c|c|}
\hline Groups & Observation group $(n=40)$ & Control group $(n=37)$ & $t$ & $P$ \\
\hline \multicolumn{5}{|l|}{ Serum PRL (ng/mL) } \\
\hline Before treatment & $70.41 \pm 15.28$ & $69.37 \pm 16.96$ & 0.283 & 0.778 \\
\hline 3 months of treatment & $14.27 \pm 3.20$ & $18.81 \pm 7.03$ & 3.693 & 0.001 \\
\hline 6 months of treatment & $6.27 \pm 1.67$ & $13.36 \pm 4.10$ & 10.075 & 0.001 \\
\hline $\mathrm{P}$ & 0.001 & 0.001 & - & - \\
\hline \multicolumn{5}{|l|}{ Serum KP (nmol/L) } \\
\hline Beforetreatment & $10.35 \pm 2.13$ & $10.40 \pm 2.34$ & 0.098 & 0.922 \\
\hline 3 months of treatment & $20.07 \pm 1.34$ & $19.12 \pm 1.85$ & 2.595 & 0.011 \\
\hline $\mathrm{P}$ & 0.001 & 0.001 & - & - \\
\hline
\end{tabular}

Italic $\mathrm{P}$ values indicate $\mathrm{P}<0.05$.

Table 3 Comparison of SAS total scores and individual item scores between the two groups $(\bar{x} \pm \mathrm{s})$

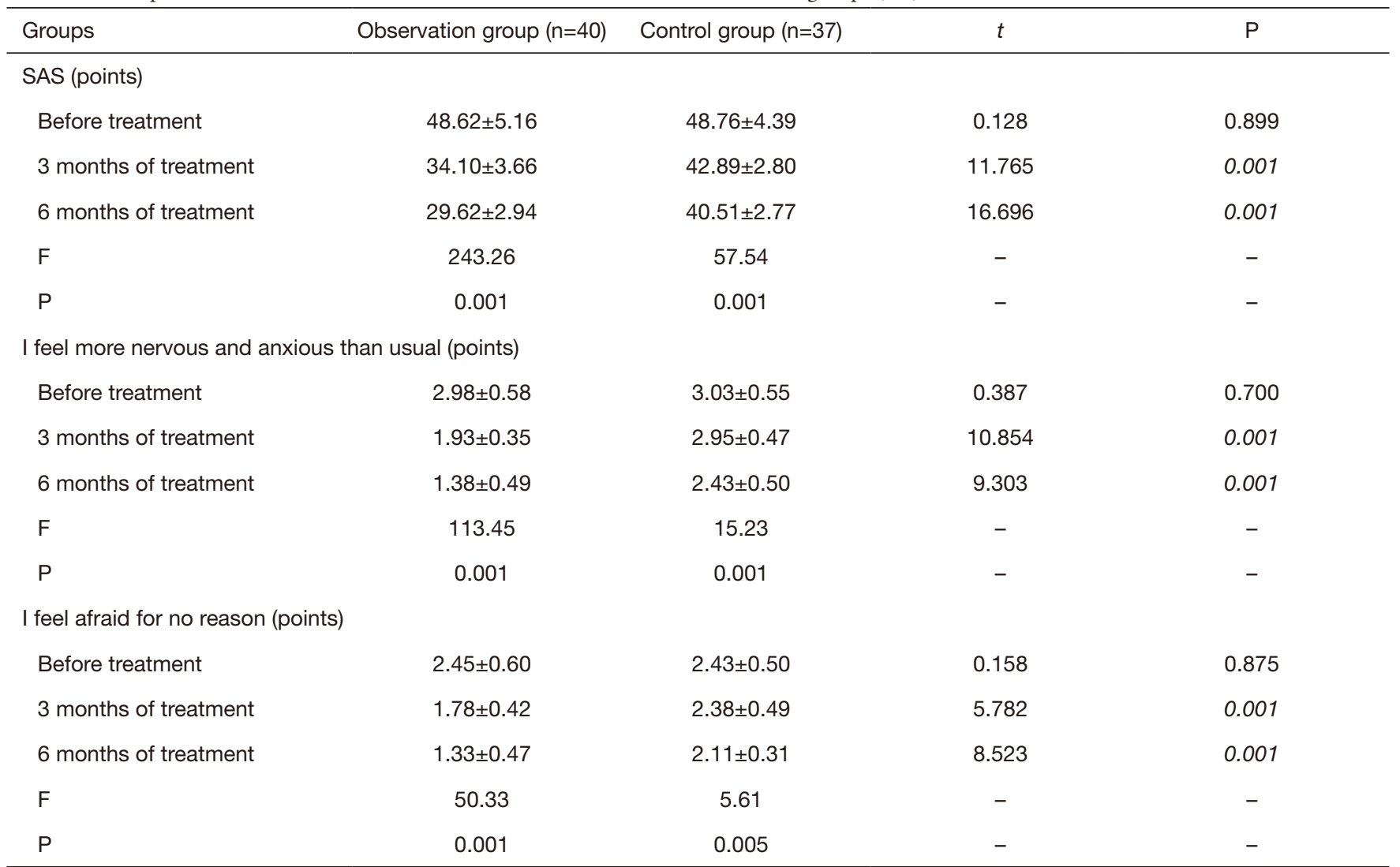

Table 3 (continued) 
Table 3 (continued)

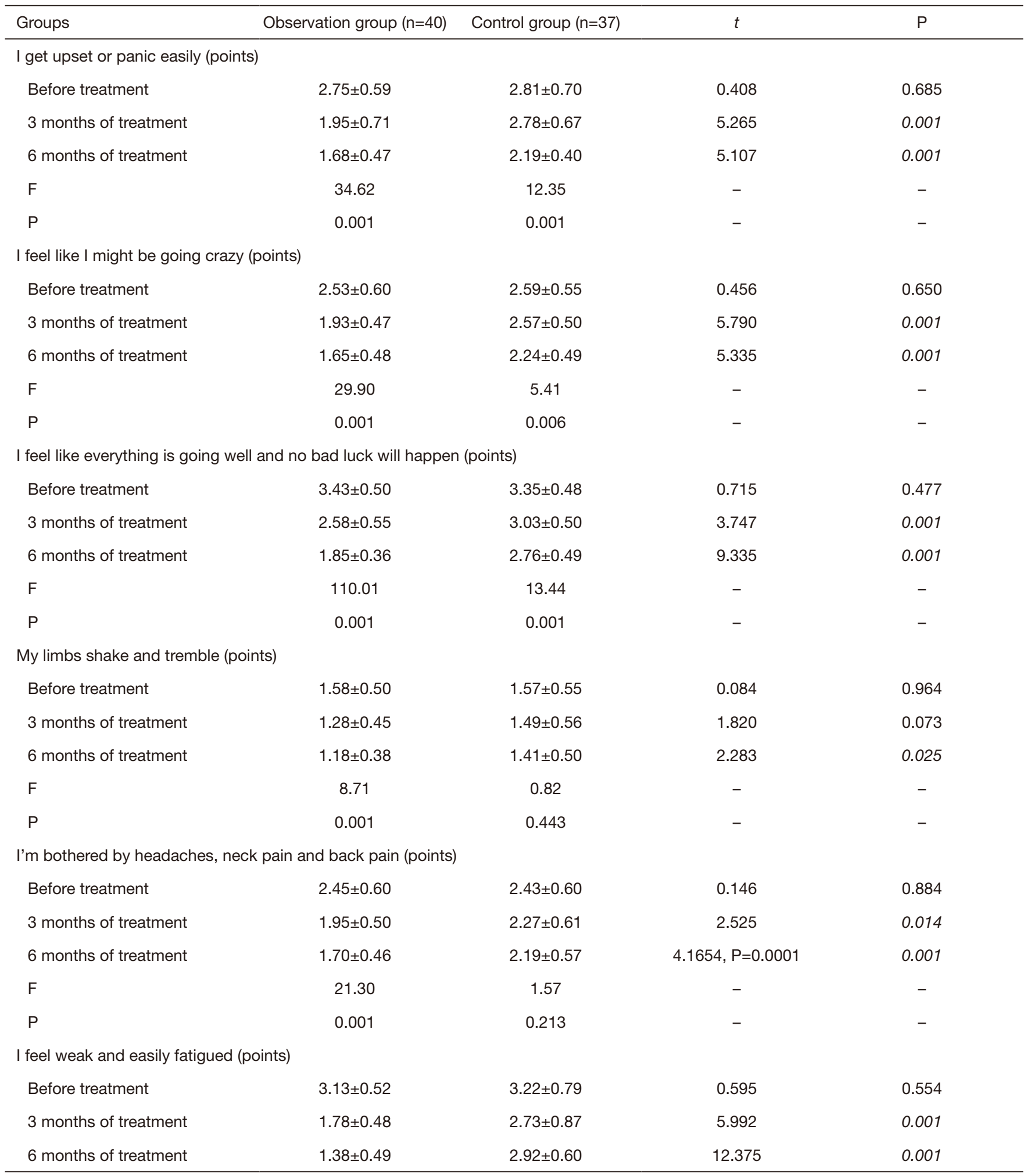

Table 3 (continued) 
Table 3 (continued)

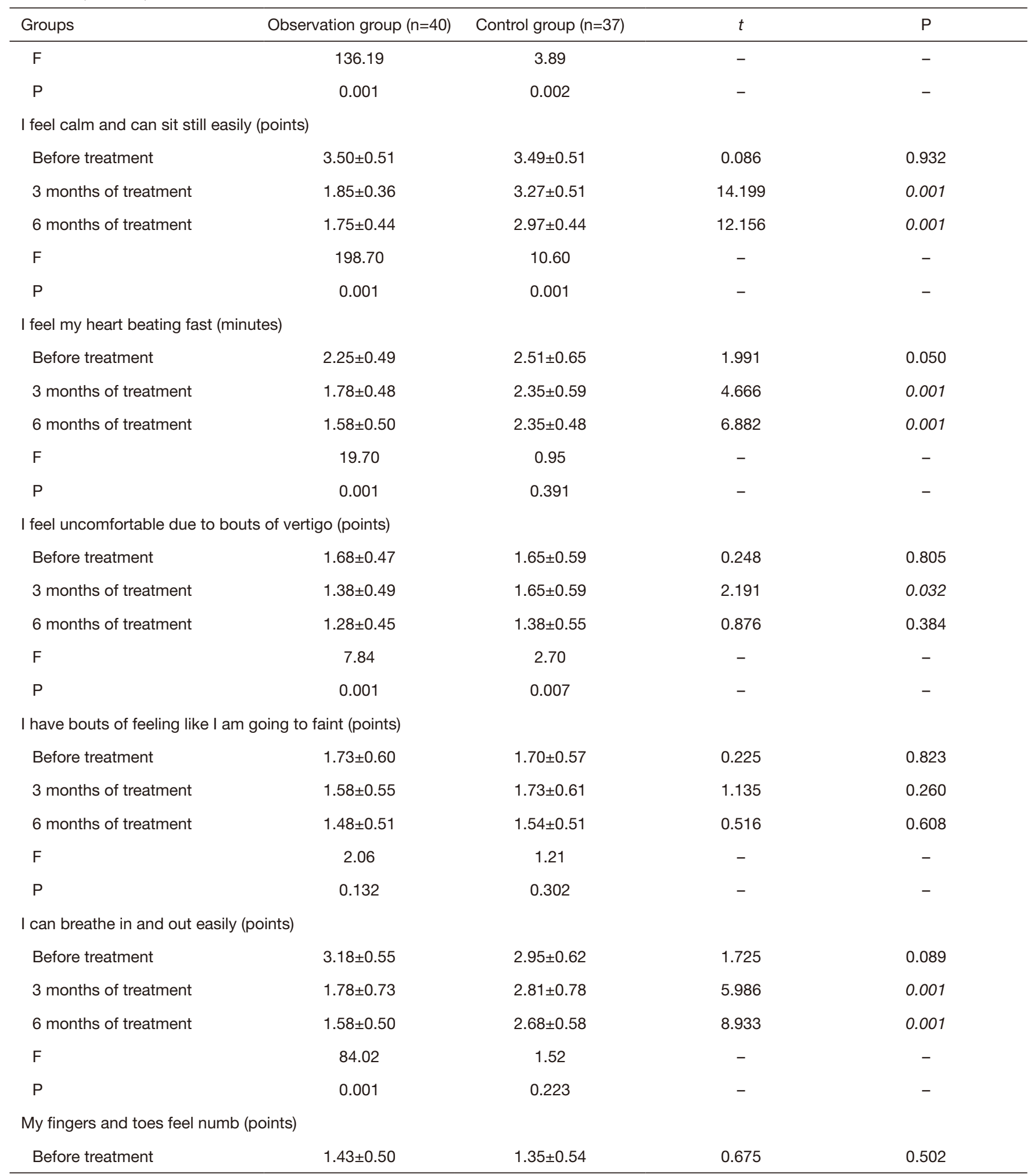

Table 3 (continued) 
Table 3 (continued)

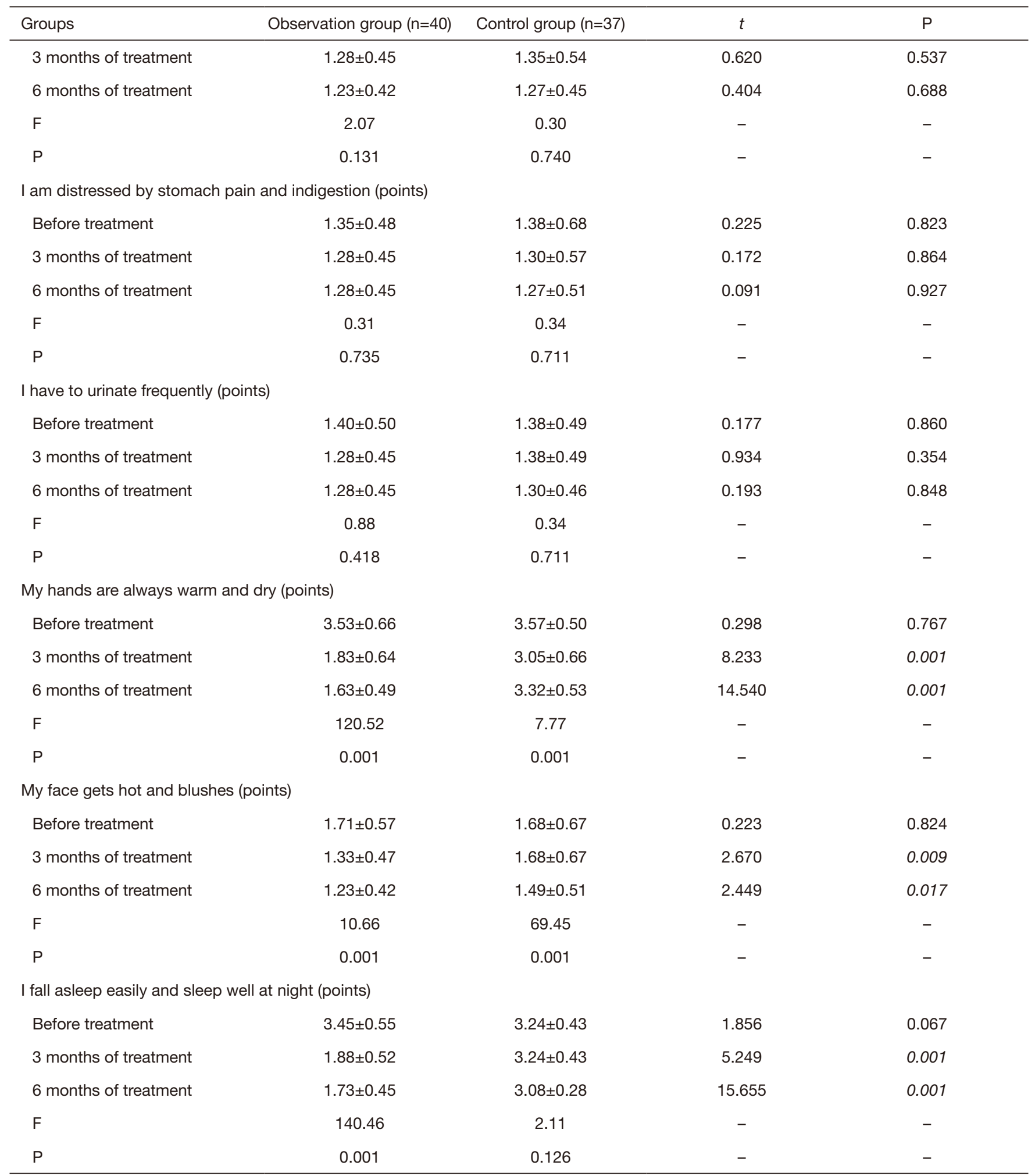

Table 3 (continued) 
Table 3 (continued)

\begin{tabular}{|c|c|c|c|c|}
\hline Groups & Observation group $(n=40)$ & Control group $(n=37)$ & $t$ & $\mathrm{P}$ \\
\hline Before treatment & $2.68 \pm 0.57$ & $2.43 \pm 0.77$ & 1.628 & 0.108 \\
\hline 3 months of treatment & $1.83 \pm 0.64$ & $2.43 \pm 0.77$ & 3.729 & 0.001 \\
\hline 6 months of treatment & $1.60 \pm 0.50$ & $2.30 \pm 0.57$ & 5.739 & 0.001 \\
\hline $\mathrm{P}$ & 0.001 & 0.662 & - & - \\
\hline
\end{tabular}

Italic $P$ values indicate $P<0.05$.

Table 4 Comparison of ISI total scores and individual item scores between the two groups $(\bar{x} \pm \mathrm{s})$

\begin{tabular}{|c|c|c|c|c|}
\hline Groups & Observation group $(n=40)$ & Control group $(\mathrm{n}=37)$ & $t$ & $\mathrm{P}$ \\
\hline Before treatment & $15.43 \pm 3.55$ & $15.08 \pm 3.12$ & 0.458 & 0.648 \\
\hline 3 months of treatment & $12.38 \pm 2.86$ & $14.03 \pm 3.17$ & 2.401 & 0.019 \\
\hline 6 months of treatment & $9.88 \pm 2.45$ & $13.41 \pm 3.28$ & 5.377 & 0.001 \\
\hline $\mathrm{P}$ & 0.001 & 0.080 & - & - \\
\hline \multicolumn{5}{|c|}{ Difficulty in falling asleep (points) } \\
\hline Before treatment & $3.28 \pm 0.60$ & $3.16 \pm 0.60$ & 0.877 & 0.383 \\
\hline 3 months of treatment & $2.05 \pm 0.55$ & $2.89 \pm 0.57$ & 6.580 & 0.001 \\
\hline$P$ & 0.001 & 0.005 & - & - \\
\hline \multicolumn{5}{|c|}{ Difficulty maintaining sleep (points) } \\
\hline Before treatment & $1.98 \pm 0.70$ & $2.08 \pm 0.55$ & 0.693 & 0.490 \\
\hline 3 months of treatment & $1.60 \pm 0.59$ & $1.95 \pm 0.47$ & 2.864 & 0.005 \\
\hline 6 months of treatment & $1.23 \pm 0.58$ & $1.81 \pm 0.46$ & 4.836 & 0.001 \\
\hline $\mathrm{F}$ & 14.37 & 2.75 & - & - \\
\hline $\mathrm{P}$ & 0.001 & 0.068 & - & - \\
\hline \multicolumn{5}{|c|}{ The problem of waking up too early (points) } \\
\hline
\end{tabular}

Table 4 (continued) 
Table 4 (continued)

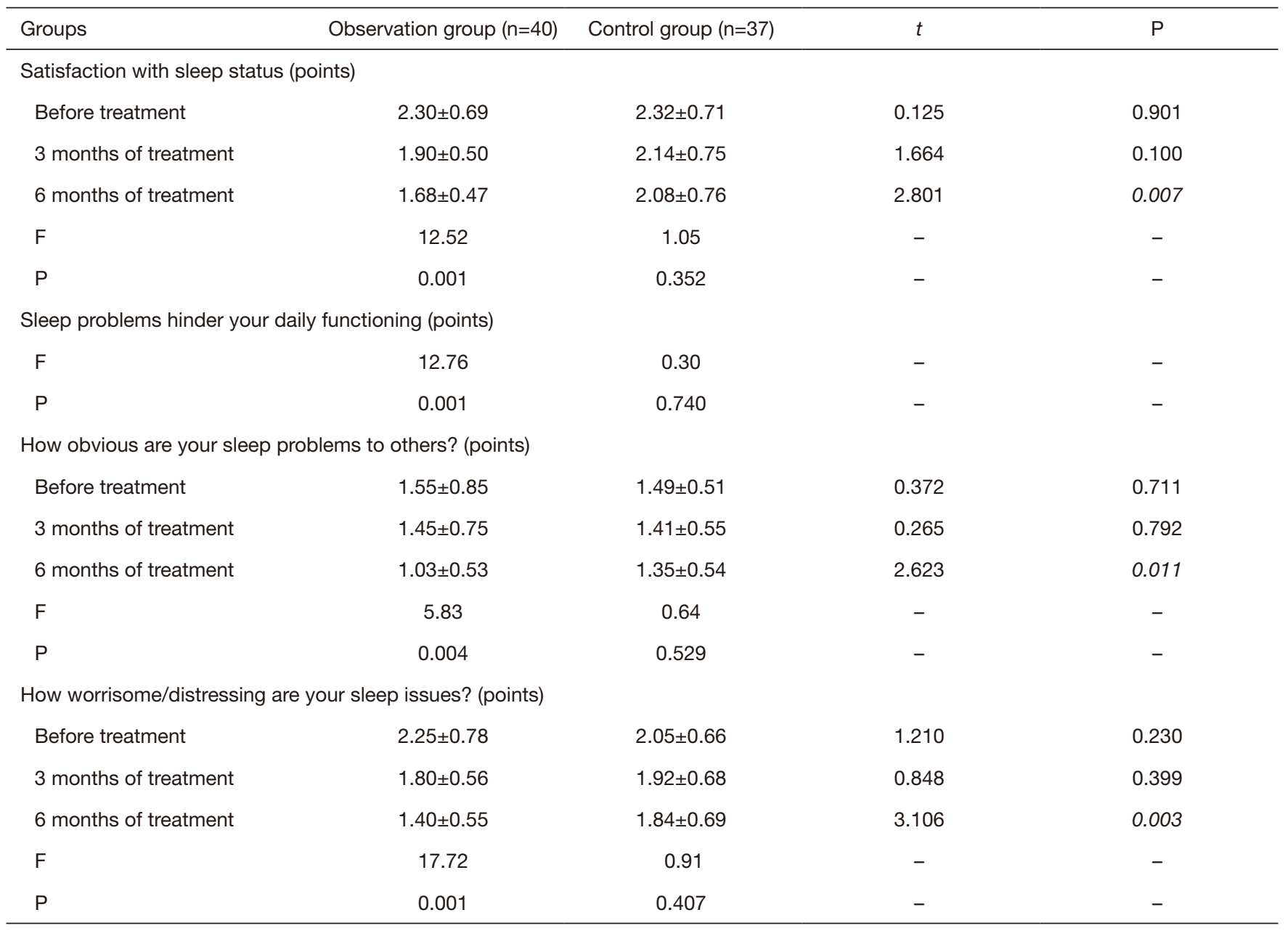

Italic $\mathrm{P}$ values indicate $\mathrm{P}<0.05$.

Table 5 Comparison of pregnancy, early miscarriage, and adverse reaction rates between the two groups [n, (\%)]

\begin{tabular}{lcccc}
\hline Groups & Number of cases & Pregnancy rate & Early miscarriage rate & Adverse reaction rate \\
\hline Observation group $(\mathrm{n}=40)$ & 40 & $25(62.50)$ & $4(10.00)$ & $3(7.50)$ \\
Control group $(\mathrm{n}=37)$ & 37 & $14(37.84)$ & $12(32.43)$ & $9(24.32)$ \\
$\chi^{2}$ & - & 4.677 & 5.876 & 4.136 \\
$P$ & - & 0.031 & 0.015 & 0.042 \\
\hline
\end{tabular}

Italic $P$ values indicate $P<0.05$.

\section{Discussion}

The mechanism of HPRL is a dysfunction of the hypothalamic-pituitary-ovarian axis (HPO), which is affected by several factors, including intracranial tumors, intracranial inflammation, primary hypothyroidism, pharmacological factors, pregnancy, and lactation. This disease has specificity (e.g., organic tissue heterogeneity), which can have a serious impact on the quality of life of patients $(12,13)$. In studies on the treatment of HPRL, 
bromocriptine is commonly used as a first-line treatment in Western medicine. For the HPRL patients with microand macroprolactinomas, dopamine agonists (DAs) can be applied to normalize of PRL secretion and gonadal function. Currently, the most commonly used DAs are bromocriptine, cabergoline, pergolide and quinagolide. However, these medicines have significant adverse side effects that can affect treatment compliance. Therefore, to enhance patient treatment compliance and improve the limitations on long-term treatment, it is necessary to combine bromocriptine with methods that have fewer adverse effects, milder side effects, and good efficacy. In this paper, we used a combination of TCM BSZY-D and bromocriptine and found that patients who received the combined treatment showed a significant improvement in clinical symptoms, which may be correlated with a more targeted approach and better efficacy when combining TCM and Western medicine treatments.

Related studies (14-16) have concluded that abnormally elevated serum PRL is a sign of HPRL. Serum PRL is typically suppressed by the hypothalamus through dopamine secretion and is stimulated by 5 -hydroxytryptamine (5-HT), but under the influence of external factors, dopamine secretion is either suppressed or $5-\mathrm{HT}$ is secreted in large amounts, resulting in an imbalance in the secretion of PRL. High PRL inhibits the pituitary gland's secretion of gonadotropins, leading to abnormal production of estrogen, follicle-stimulating hormone (FSH), and luteinizing hormone, preventing healthy follicle discharge or development. In turn, this can cause irregular menstruation, galactorrhea, and clinical symptoms of infertility. In addition, high prolactin causes negative feedback damage to estrogen, which prevents progesterone synthesis and leads to a thin endometrium, increasing the rate of miscarriage. Western medicine commonly uses bromocriptine as the drug of choice for high serum PRL treatment. Bromocriptine inhibits the secretory activity of prolactin cells by activating the dopamine-like effect, thereby reducing the serum PLR of patients (17). In the present study, serum PRL decreased significantly in both groups of patients. For HPRL with non-neurological diseases and tumor compression, when serum PRL decreases, HPO function is gradually restored, enabling hypothalamic kisspeptin expression to normalize the hypothalamic-pituitary-gonadal (HPG) axis $(18,19)$ Serum KP is a peptide hormone widely distributed in the brain and multiple tissues and regulates cancer cells, reproductive function, and endocrine function. Kisspeptin regulates brain regions associated with individual behavior, and kisspeptin is a possible target of psychosocial stress that inhibits LH (luteinizing hormone) impulses, while the regulation of serum KP can stimulate the secretion of LH and the FSH (20). BSZY-D can increase serum KP expression by affecting widespread prolactin receptors in hypothalamus GT1-7 cells. In turn, this allows regulation of serum hormone (serum LH, FSH, PRL, estrogen, progesterone, etc.) levels and improves ovulation, thus treating infertility and early miscarriage $(21,22)$.

Bromocriptine has significant efficacy in HPRL, but several studies have shown that long-term continuous use of bromocriptine can produce serious adverse effects $(23,24)$. Long-term DA administration can have unwanted psychological side effects, limiting follow-up treatment of patients and negatively affecting their sleep quality. Previous studies provide evidence to support the use of DAs in reducing prolactin levels and persistent HPRL, such as cabergoline $(25,26)$. However, some patients are resistant or intolerant to DAs. Therefore, it is necessary to seek an alternative approach with fewer side effects and more effectiveness. The symptoms of HPRL are highly similar to those of "galactorrhea disease", "infertility", and "amenorrhea" in TCM, which are considered to be symptoms of spleen and kidney deficiency, and liver depression. The patient's inability to have children may lead to depression, resulting in emotional disorders. This can cause patients with Qi and blood disorder, blood does not follow the regular path of xuehai for menstruation, but with liver Qi on the inverse breast for galactorrhea, resulting in high prolactin hyperemia, galactorrhea, menstruation, amenorrhea or infertility. The formula used in the observation group is suitable for those with kidney deficiency-type infertility. In the formula, the primary component is deer horn slices, which have the effect of tonifying the kidney and nourishing Yang. The secondary components are violet quartz, fried white peony root, cuscutae semen, dioscoreae rhizoma, and corni fructus, of which violet quartz calms the heart and tranquilizes the mind, warming both the lung and the uterus. The other four drugs are commonly used to tonify the kidneys, together with radix bupleuri to detoxify the liver and relieve depression, gambir plant to calm the heart and tranquilize the mind, dens draconis to treat palpitations, body heat, and heart trouble, and purple salvia miltiorrhiza to nourish the blood and calm the mind. The whole decoction is well formulated and soothes the heart, liver, and qi. It also nourishes the liver and kidney, strengthens the spleen, and 
enriches the blood, meeting HPRL's therapeutic needs $(27,28)$. Several studies have reported the effective effect of TCM on HPRL treatment. A pilot randomized controlled trial on 56 women with idiopathic HPRL showed a role for chamomile in the modulation of prolactin secretion in women by acting on dopamine receptors (29). Besides, the plant species of the Verbenaceae family have been confirmed to play a role in the treatment of gynecological diseases and premenstrual symptoms, such as depression, HPRL and dysmenorrhea (30).

In this study, the overall clinical efficacy in the observation group was superior to that in the control group. Our findings suggested that adding purple fluoritum, gambir plant, and dens draconis in the BSZY-D effectively calmed the mind and resolved insomnia and poor sleep, resulting in significantly lower SAS and ISI scores in the observation group than in the control group. In addition, modern pharmacology has shown that the neutral saponin component of the triple mushroom class in radix bupleuri can regulate the nervous system, and the total glucosides of peonia in white peony can regulate the hypothalamicpituitary-adrenal axis and enhance immune function, which improves serum PRL and serum KP levels. The sustained decrease in SAS and ISI scores in both groups was also associated with improvement in HPRL. High serum PRL occurs when dopamine secretion decreases, and therefore the administration of bromocriptine produces a dopaminelike effect in patients resulting in improved depressive symptoms and sleep quality (31-33). However, it should be noted that six individual items on the SAS ("I have to urinate frequently", "I am distressed by stomach pain and indigestion", "my fingers and toes feel numb", "I have bouts of feeling like I am going to faint", "I feel uncomfortable due to bouts of vertigo", and "my limbs shake and tremble"), were not statistically significant between the two groups at 3 and 6 months of treatment, which may be related to the fact that specific limbs and urinary organ tissues were less affected and the adverse effects caused by bromocriptine were mild. On the ISI score, at 3 months of treatment, the observation group scored significantly lower than the control group on two items (difficulty in falling asleep and difficulty in maintaining sleep), and at 6 months of treatment, there was a significant difference in six items (the exception being the problem of waking up too early). The analysis suggests that the 6-month results may be related to the alleviation of bromocriptine side effects after the combined application of BSZY-D, while difficulty in falling asleep and maintaining sleep was more serious before treatment and was alleviated more rapidly. While the pregnancy rate was significantly higher in the observation group, the early miscarriage rate was significantly lower than that in the control group due to a greater reduction in serum PRL and more attenuation of estrogenic negative feedback damage. These findings suggest that the lower rate of adverse effects in the observation group may be related to the fact that herbal treatment allowed a reduction in the dosage of bromocriptine $(34,35)$.

The limitations of this study include the small number of patients included in the study and the exclusion of patients with elevated PRL due to pituitary tumors and other organic heterogeneities, which may have led to some bias in the study results. Therefore, more studies with larger sample sizes are needed to verify the efficacy of the combination of bromocriptine with BSZY-D.

In conclusion, bromocriptine combined with BSZY-D has a good therapeutic effect on HPRL and infertility due to HPRL. The combined treatment is superior to bromocriptine alone in improving patients' sleep and emotional symptoms and has potential for future treatment efficacy.

\section{Acknowledgments}

Funding: None.

\section{Footnote}

Reporting Checklist: The authors have completed the STROBE reporting checklist. Available at https://dx.doi. org/10.21037/apm-21-3111

Data Sharing Statement: Available at https://dx.doi. org/10.21037/apm-21-3111

Conflicts of Interest: All authors have completed the ICMJE uniform disclosure form (available at https://dx.doi. org/10.21037/apm-21-3111). The authors have no conflicts of interest to declare.

Ethical Statement: The authors are accountable for all aspects of the work in ensuring that questions related to the accuracy or integrity of any part of the work are appropriately investigated and resolved. All procedures performed in this study involving human participants were in accordance with the Declaration of Helsinki (as revised in 2013). The study was approved by the Ethics Committee 
of Jiangsu Provincial Hospital of Traditional Chinese Medicine (also named Affiliated Hospital of Nanjing University of Chinese Medicine, No. 2108NL-030-02) and informed consent was taken from all the patients.

Open Access Statement: This is an Open Access article distributed in accordance with the Creative Commons Attribution-NonCommercial-NoDerivs 4.0 International License (CC BY-NC-ND 4.0), which permits the noncommercial replication and distribution of the article with the strict proviso that no changes or edits are made and the original work is properly cited (including links to both the formal publication through the relevant DOI and the license). See: https://creativecommons.org/licenses/by-nc-nd/4.0/.

\section{References}

1. Fachi MM, de Deus Bueno L, de Oliveira DC, et al. Efficacy and safety in the treatment of hyperprolactinemia: A systematic review and network meta-analysis. J Clin Pharm Ther 2021. [Epub ahead of print]. doi: 10.1111/ jcpt. 13460.

2. Villanueva DL, Evangelista LK, Espanillo-Villanueva C, et al. Use of bromocriptine for the treatment of peripartum cardiomyopathy: a meta-analysis of randomized controlled trials. Cardiology and Cardiovascular Research 2020;4:52.

3. Zhao Y, Han Y, Song J, et al. Comparison of different therapeutic methods of bromocriptine in treating female hyperprolactinemia. Systems Medicine 2018. Available online: http://en.cnki.com.cn/Article_en/CJFDTotalXTYX201803038.htm

4. Li J. Efficacy of combining clomiphene with BSZY-D in the treatment of ovulatory disorders of infertility. Clinical Research in Traditional Chinese Medicine 2018;10:76-8.

5. Yin $Y$, Wang $M$, Pan $Y$, et al. Clinical study of 32 cases of kidney deficiency-type polycystic ovary syndrome infertility treated with BSZY-D combined with western medicine. Zhejiang Journal of Traditional Chinese Medicine 2017;52:167-8.

6. Vilar L, Abucham J, Albuquerque JL, et al. Controversial issues in the management of hyperprolactinemia and prolactinomas - An overview by the Neuroendocrinology Department of the Brazilian Society of Endocrinology and Metabolism. Arch Endocrinol Metab 2018;62:236-63.

7. Wang H, Liu A, Wang X. Efficacy Analysis of Using Yigan Tiaoshen Decoction Combined with Time Acupuncture in the Treatment of Idiopathic Infertility with High Prolactin. Journal of Sichuan of Traditional Chinese
Medicine 2018. Available online: http://en.cnki.com.cn/ Article_en/CJFDTotal-SCZY201812058.htm

8. Wang J, Xiang-Yan R, Yin DM, et al. Clinical Study of Female Sex Hormones Hyperprolactinemia Bromocriptine Ovulation Different Dosing Egimens. Progress in Modern Biomedicine 2017. Available online: https://xueshu.baidu.com/usercenter/paper/show?pape rid=0bf797ff 2 aeb3c1ba725553227307d8f\&site=xueshu se\&hitarticle $=1$

9. Liang L, Wang L, He Q, et al. Secondary resistance to bromocriptine and cabergoline therapy in hyperprolactinemia: One case of pregnancy with IVF-ET. Available online: https://www.researchgate. net/publication/341675329_Secondary_resistance_ to_bromocriptine_and_cabergoline_therapy_in_ hyperprolactinemia_One_case_of_pregnancy_with_ IVF-ET

10. Setyowati A, Chung MH, Yusuf A. Development of selfreport assessment tool for anxiety among adolescents: Indonesian version of the Zung self-rating anxiety scale. Journal of Public Health in Africa 2019;10. Available online: https://www.researchgate.net/ publication/336934111_Development_of_self-report_ assessment_tool_for_anxiety_among_adolescents_ Indonesian_version_of_the_Zung_self-rating_anxiety_ scale

11. Schulte T, Hofmeister D, Mehnert-Theuerkauf A, et al. Assessment of sleep problems with the Insomnia Severity Index (ISI) and the sleep item of the Patient Health Questionnaire (PHQ-9) in cancer patients. Support Care Cancer 2021. [Epub ahead of print]. doi: 10.1007/s00520021-06282-x.

12. Huan YU, Qian Y. Analysis of Correlation between Serum Reproductive Hormone Levels and Chinese Medicine Syndrome Types in Patients with Polycystic Ovary Syndrome. Journal of New Chinese Medicine 2019. Available online: http://en.cnki.com.cn/Article_en/ CJFDTotal-REND201908008.htm

13. Sokhadze K, Kvaliashvili S, Kristesashvili J. Reproductive function and pregnancy outcomes in women treated for idiopathic hyperprolactinemia: A non-randomized controlled study. Int J Reprod Biomed 2020;18:1039-48.

14. Wu YP, Wang YB, Wu ZB. Bromocriptine-responsive supersellar germinoma with the expression of dopamine receptors: a case report. Clin Neurol Neurosurg 2019;176:15-8.

15. Gonzaga MFM, de Castro LF, Naves LA, et al. Prolactinomas resistant to treatment with dopamine 
agonists: long-term follow-up of six cases. Front

Endocrinol (Lausanne) 2018;9:625.

16. Lianfang QI. ChaiYu JiSheng Decoction Combined with Bromocriptine in Treating 45 Cases of Hyperprolactinemia-induced Delayed Menstrual Cycle. Western Journal of Traditional Chinese Medicine 2018. Available online: http://en.cnki.com.cn/Article_en/ CJFDTotal-GSZY201808030.htm

17. Valdes CT, Schutt A, Simon C. Implantation failure of endometrial origin: it is not pathology, but our failure to synchronize the developing embryo with a receptive endometrium. Fertil Steril 2017;108:15-8.

18. Yang JA, Song CI, Hughes JK, et al. Acute Psychosocial Stress Inhibits LH Pulsatility and Kiss1 Neuronal Activation in Female Mice. Endocrinology 2017;158:3716-23.

19. Acevedo-Rodriguez A, Kauffman AS, Cherrington BD, et al. Emerging insights into hypothalamic-pituitary-gonadal axis regulation and interaction with stress signalling. J Neuroendocrinol 2018;30:e12590.

20. Jiang $X$, Zhou H, Shi M, et al. Bu-shen-zhu-yun decoction promotes synthesis and secretion of FSH $\beta$ and $\mathrm{LH} \beta$ in anterior pituitary cells in vitro. Biomed Pharmacother 2018;102:494-501.

21. Zhou BR, Zhou HF, Liu B, et al. Effects of Bu-Shen-ZhuYun Decoction on pituitary $\mathrm{GnRH}$ receptor signaling system in LPD model rats. Journal of Nanjing University of Traditional Chinese Medicine 2017;33:554-9.

22. Zhou BR, Dai JX, Yang LJ, et al. Effects of Bu-Shen-ZhuYun Decoction on gonadotropin and its key regulatory factors in luteal insufficiency rats. Journal of Graduate Medical Science 2016;29:1153-7.

23. Capani F, Quarracino C, Caccuri R, et al. Astrocytes As the Main Players in Primary Degenerative Disorders of the Human Central Nervous System. Front Aging Neurosci 2016;8:45.

24. Krysiak R, Szkróbka W, Okopień B. The effect of bromocriptine treatment on sexual functioning and depressive symptoms in women with mild hyperprolactinemia. Pharmacol Rep 2018;70:227-32.

25. Wang AT, Mullan RJ, Lane MA, et al. Treatment of hyperprolactinemia: a systematic review and meta-analysis. Syst Rev 2012;1:33.

26. Chen H, Fu J, Huang W. Dopamine agonists for preventing future miscarriage in women with idiopathic hyperprolactinemia and recurrent miscarriage history. Cochrane Database Syst Rev 2016;7:CD008883.

27. Tian Y, Gao X, Yang D, et al. Effect of Sanjie Quyu decoction combined with bromocriptine on hepatic stagnation hyperprolactinemia and peripheral blood lymphocyte subsets and cortisol levels. Modern Journal of Integrated Traditional Chinese and Western Medicine 2018. Available online: http://en.cnki.com.cn/Article_en/ CJFDTOTAL-XDJH201823010.htm

28. Zhang CH, Ma K, Yuan BC, et al. Bushen Huoxue herbal medicine for treating hyperprolactinemia in women: a Meta-analysis. Zhongguo Zhong Yao Za Zhi 2019;44:1087-93.

29. Kabiri M, Kamalinejad M, Bioos S, et al. Comparative Study of the Effects of Chamomile (Matricaria Chamomilla L.) and Cabergoline on Idiopathic Hyperprolactinemia: A Pilot Randomized Controlled Trial. Iran J Pharm Res 2019;18:1612-21.

30. Akbaribazm M, Goodarzi N, Rahimi M. Female infertility and herbal medicine: An overview of the new findings. Food Sci Nutr 2021;9:5869-82.

31. Peng Y, Su Y, Jiang Y. Effect of the warming and tonifying kidney- yang recipe on monoamine neurotransmitters and pathological morphology of hippocampus tissue in depression model rats. Technol Health Care 2020;28:237-44.

32. Celik O, Celik N, Aydin S, et al. Ghrelin action on GnRH neurons and pituitary gonadotropes might be mediated by GnIH-GPR147 system. Horm Mol Biol Clin Investig 2016;25:121-8.

33. Mittelman-Smith MA, Wong AM, Micevych PE. Estrogen and Progesterone Integration in an in vitro Model of RP3V Kisspeptin Neurons. Neuroendocrinology 2018;106:101-15.

34. Chen P, Chen L. Effect of Bromocriptine on Gonadotropin-induced Ovulation in Women with Hyperprolactinemia. Medical Information 2018. Available online: http://en.cnki.com.cn/Article_en/CJFDTotalYXXX201802046.htm

35. Lingxia QI, Zhang X. Professor Zhang Xiaosu's Experience in Treating Hyperprolactinemia. Western Journal of Traditional Chinese Medicine 2019. Available online: http://en.cnki.com.cn/Article_en/CJFDTotalGSZY201907013.htm

Cite this article as: Feng $\mathrm{H}$, Zhong $\mathrm{Q}$, Zhou H, Jiang $\mathrm{X}$, Ding Y. The effects of combined bromocriptine and Bu-shenzhu-yun decoction on serum hormones, anxiety, and pregnancy in hyperprolactinemic infertility patients. Ann Palliat Med 2021;10(12):12230-12243. doi: 10.21037/apm-21-3111 